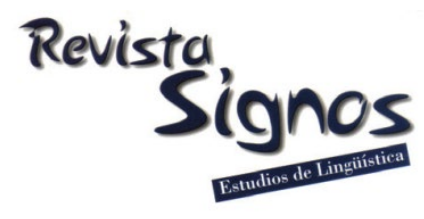

\title{
Desde lo subléxico hasta lo sintáctico: Reasunción, sustantivos relacionales y sustantivos de parte-todo*
}

\author{
From sublexical features to syntax: Resumption, relational \\ nouns and part-whole nouns
}

\author{
Jorge Agulló \\ UNIVERSIDAD AUTÓNOMA DE MADRID \\ ESPAÑA \\ jorge.agullo@uam.es
}

Recibido: 05-VII-2018 / Aceptado: 26-XII-2018

DOI: $10.4067 /$ S0718-09342019000200590

\section{Resumen}

El objetivo de este artículo es determinar qué clases de sustantivos pueden formar parte de la reasunción en español, cuáles son sus propiedades subléxicas y de qué forma se establece la relación de concordancia entre el sustantivo reasuntivo y el antecedente de la cláusula de relativo. Asumido el continuum categorial de la reasunción, los sustantivos relacionales y de parte-todo se analizan en términos de la Teoría del Lexicón Generativo de Pustejovsky (1991, 1995, 1998): las representaciones subléxicas, estructuralmente jerárquicas, acogen una información que adscribe el sustantivo a una de las clases y le permite operar como reasuntivo si su estructura de Qualia habilita una relación de concordancia con el antecedente. Se establecen ciertas diferencias entre las dos clases de sustantivos, se discuten algunos datos sobre el comportamiento de cada clase y se esbozan propuestas de representaciones léxicas con la finalidad de incardinar en la teoría lexicológica una explicación sobre la información codificada en la entrada léxica del sustantivo que faculta la reasunción en la sintaxis del español.

Palabras Clave: Reasunción, lexicón generativo, estructura subléxica, nombres relacionales, nombres de parte-todo. 


\begin{abstract}
The aim of this paper is to determine which noun classes may function as resumptives in Spanish syntax, which are their lexical properties and in which way the relation is established between the resumptive noun and the head of the relative clause. Assuming that resumption may involve several categories, relational and part-whole nouns are analyzed in terms of the Generative Lexicon Theory put forth in Pustejovsky (1991, 1995, 1998): lexical representations, hierarchically structured, account for the information which classifies the noun as either relational or part-whole denoting and which enables the noun to function as resumptive if its Qualia Structure allows for an agreement relation with the antecedent. Similarities between the two noun classes are established, some data is presented regarding their syntactic behaviour and contextsensitive lexical representations are proposed in order to provide a theoretical explanation of the information allegedly encoded within the lexical entry of the noun, which enables nominal resumption in Spanish syntax.
\end{abstract}

Key Words: Resumption, generative lexicon, lexical structure, relational nouns, partwhole nouns.

\title{
INTRODUCCIÓN
}

La sintaxis del español atestigua un fenómeno, denominado 'reasunción', por el que las cláusulas de relativo, bajo ciertas circunstancias, habilitan una copia o refuerzo pronominal, de naturaleza tónica o átona, que ocupa lo que se ha denominado 'posición relativizada' (Keenan \& Comrie, 1977; Keenan, 1985; Andrews, 2007; Sanfelici \& Poletto, 2017; entre otros), esto es, la posición reservada a la variable de interpretación ligada, en el sentido de Chomsky (1981), en las cláusulas de relativo. El pronombre reasuntivo asume (a) la función sintáctica, (b) la referencia y (c) las marcas de caso, propiedades que corresponden al pronombre relativo en la estrategia canónica. Las muestras dialectales de (1) ilustran someramente el fenómeno de la reasunción pronominal en español, que involucra tanto pronombres reasuntivos átonos (1a) como tónicos, en función de sujeto (1b) y objeto oblicuo (1c) ${ }^{1}$ :

(1) a. La escuela la hicieron ahí, una esçuẹela que la están haciendo unos de Tolosa aquí (COSER, Turiso [Álava]).

b. Y había quien podía vender a algún vecino que no tendría vacas él, ¿verdad? (COSER, Cossío [Cantabria]).

c. Si tiene un ganano que vive de ello y una fiera se lo come, usted qué hace. Eso muy mal (COSER, Linares [Cantabria]).

El panorama de estudios consagrados a este fenómeno evidencia denominaciones muy diversas: estrategia del pronombre 'reasuntivo' (Comrie \& Keenan, 1979; Engdahl, 1985; Prince, 1990; Andrews, 2007), del pronombre 'anafórico' (Givón, 1975, 1979, 2001; Maxwell, 1979) o 'de retención del pronombre' (Keenan, 1972; Keenan \& Comrie, 1977, 1979; Comrie, 1981, 2004), a las que gramáticos del español han añadido la de 'despronominalización' (Lope Blanch, 1986, 2001) y la de 
construcciones de relativo 'de retoma' (Bentivoglio, 2003; Cerrón-Palomino, 2006; entre otros). El fenómeno ha sido ampliamente documentado para las lenguas románicas (Stark, 2009). La teoría sintáctica ha puesto de manifiesto que ciertas lenguas, como el inglés, pueden habilitar adjetivos reasuntivos (2), lo que permite añadir la oración atestiguada en español de (3) a las muestras de (1):

(2) There was one prisoner who we couldn't even figure out why the poor guy was even in jail [ejemplo tomado de Kroch, 1981:129].

(3) Siempre suele haber algún tipo un poco, eh, que es fuera de serie. Aquí había un tipon, que ya murió el pobre, le pilló un camión (COSER, Gabiria [Guipúzcoa]).

El continuum categorial de la reasunción no encuentra, sin embargo, su límite en los adjetivos. Así, la información pronominal que transmite la flexión verbal (4), en lenguas como el irlandés (McCloskey \& Hale, 1984) se ha revelado categoría productiva de función reasuntiva:

(4) na daoine a $\mathrm{N}$ raibh mé ag dúil go $\mathrm{N}$ gcuirfidis isteach

the people comp was I expect prog comp put.cond.3pl in

ar an phost sin

on that job

'la gente que yo esperaba (que ellos) optarían al trabajo'

[ejemplo tomado de McCloskey \& Hale, 1984:498].

Así pues, además de pronombres, adjetivos y determinadas marcas flexivas, los sintagmas determinantes pueden participar también de la reasunción. El español, en este sentido, recurre con cierta sistematicidad a la pauta ilustrada en (5), que constituye el objeto de estudio de este artículo:

(5) a. Tengo otra hija casada, que el marido trabaja de peón de albañil, la mayor [COSER, Portillo de Solórzano (Cantabria)].

b. I1: [...] En la chopera esa es donde hacemos la comida que llamamos "la comida de la chopera" es donde hacemos la comida de la [a] [aque yo hasta ahora he sido la tesorera [COSER, Santa Eulalia de Tábara (Moreruela de Tábara, Zamora)].

En los ejemplos de (5), un SD contrae con el antecedente de la cláusula una relación de parentesco (5a) o inclusión (5b), cada una de ellas con sus respectivas propiedades subléxicas. Este artículo abordará, pues, la estrategia de relativización del 'poseedor', en un sentido laxo, que, en la Jerarquía de Accesibilidad de Keenan y Comrie (1977), constituye una de las posiciones menos accesibles a los mecanismos de construcción de oraciones de relativo (Cristofaro \& Giacalone Ramat, 2007; Stark, 2016). El objetivo es, así, dar cuenta de los procedimientos por los que la lengua española habilita la presencia de SDs reasuntivos dotados de ciertos rasgos subléxicos 
que, por un lado, legitiman la aparición de dichos sintagmas y, por otro, explican la agramaticalidad de los SDs desprovistos de tales rasgos subléxicos. Para ello, asumo la Teoría del Lexicón Generativo desarrollada por Pustejovsky $(1991,1995,1998)$.

El artículo, con tal fin, se estructura de la siguiente forma: sobre la base empírica de la documentación del fenómeno (apartado 1), la introducción al marco teórico (apartado 2) permite el esbozo de la propuesta (apartado 3) en términos de rasgos subléxicos, aportación que cierran las conclusiones.

\section{Primera aproximación a los datos: Documentación del fenómeno}

El Corpus Oral y Sonoro del Español Rural (COSER) (Fernández-Ordóñez, 2007) me ha permitido documentar 383 ejemplos de construcciones de relativo con constituyente reasuntivo en el área septentrional de la Península, esto es, desde la franja gallego-astur-cántabra hasta la provincia de Lérida, pasando por las regiones subpirenaicas. Sin embargo, sobre el total de ocurrencias, tan solo ocho $(2,1 \%)$ pueden concebirse como ejemplos inequívocos de reasunción nominal, excluidas ciertas construcciones, como las causales de la enunciación o las oraciones consecutivas.

Las formas de reasunción aquí sometidas a estudio se ilustran por medio de los constituyentes sintácticos destacados de (6):

(6) a. Y habían las dos o tres casas más ricas del pueblo, dos casasas, pues, que sus hijos los llevaban incluso a la universidad de Barcelona [COSER, García (Tarragona)].

b. Tengo otrra hiją casada, que el marido trabaja de peón de albañil, la mayor [COSER, Portillo de Solórzano (Cantabria)].

c. E: ¿Cuánto tiempo amamantaban a los niños?

I2: Según como sería, había madradres que la leche no valía pa nada y le tenía que quitar su madre el pecho [COSER, Berganzo (Álava)].

d. E: ¿Y si la mujer no tenía leche?

I1: Pues tenía que coger, claro, darle a lo mejor a otra chicica que se había muerto el niño o ella le había quitao la mama y le daba de mamar [COSER, Puente de Almuhey (León)].

e. E: ¿Nietos?

I1: Tengo dos, el casao tiene otra hija y essta que murió la madre, tiene la hija que está en Madrid. Tiene dieciocho años [COSER, Santa María del Río (León)].

A pesar de que, a lo largo del artículo, se derivarán ejemplos ad hoc, las muestras dialectales de (6) evidencian que el fenómeno puede atestiguarse en distintas variedades del español peninsular, para las que el COSER constituye una fuente de estudio primordial (Fernández-Ordóñez, 2007, 2009, 2010, 2011; De Benito Moreno, 
Pueyo \& Fernández-Ordóñez, 2016). Los ejemplos de (6) evidencian que la relación de posesión-inclusión puede expresarse por medio de tres elementos reasuntivos: el determinante posesivo 'su', el artículo definido con valor de posesión inalienable y, además, el sintagma preposicional 'de él' ('del') (y vv.) en función de complemento del nombre (Brucart, 1999). Si bien la denominación de 'quesuismo' es relativamente reciente en las gramáticas, estas construcciones pueden remontarse a la lengua medieval; para más detalles sobre el fenómeno en la historia del español, véase Sánchez Lancis (2016).

\section{Introducción a un modelo dinámico del léxico: La Teoría del Lexicón Generativo}

A partir de una concepción eminentemente generativa y, por tanto, computacional del lenguaje natural, la Teoría del Lexicón Generativo, desarrollada por Pustejovsky en diversos trabajos $(1991,1995,1998,2013)$, reacciona contra el estatismo de las teorías semánticas al uso a mediados del siglo $\mathrm{xx}$, en tanto que aboga por la naturaleza dinámica del lenguaje; en este sentido: "The difficulty here for semantics and computational lexicons is that word sense enumeration cannot characterize all the possible meanings of the lexical item in the lexicon" (Pustejovsky, 1998: 46; Piera, 2009; Pustejovsky, Bouillon, Isahara, Kanzaki \& Lee, 2013).

Así concebida, la Teoría del Lexicón Generativo surge como una semántica léxica que debe asignar significados a las expresiones de forma recursiva. Con este fin, la composicionalidad débil (weak compositionality) se sustituye en Pustejovsky (1995, 1998) por una composicionalidad fuerte, en virtud de la cual el carácter generativo recae en los procesos de composición ${ }^{2}$, por los que los ítems léxicos adquieren nuevos matices de significado en el contexto $^{3}$ (Pustejovsky, 2013). En rigor, uno de los postulados más firmes de los albores de la teoría es el de que el Lexicón Generativo ahonda en los mecanismos generativos que explican los múltiples sentidos de un ítem y el uso flexible que a ellos se asocia (Anick \& Bergler, 1991; De Miguel, 2012).

Una de las principales novedades de la Teoría del Lexicón Generativo radica en dotar de distintos 'parámetros' o 'dimensiones' de interpretación a cada ítem léxico ${ }^{4}$, asimismo denominados forms o templates (Pustejovsky, 1998). Así, el contenido semántico de cada ítem léxico se estructura en cuatro niveles de representación, cuya traducción al castellano tomo de De Miguel (2009): (a) la estructura argumental, (b) la estructura eventiva, (c) la estructura de Qualia y (d) la estructura de tipificación léxica. Diversos mecanismos generativos, como la co-composición y la coacción de tipo (ingl. type coercion), operan sobre estos cuatro niveles para dotar a cada ítem léxico de nuevas interpretaciones en el contexto; esto es, constituyen un conjunto de recursos generativos que relacionan "these four levels, providing for the compositional interpretations of words in context" (Pustejovsky, 1998: 58)5. Este conjunto de hipótesis sobre la estructura del léxico me ha permitido proponer un marco en el que 
las instrucciones codificadas en las estructuras subléxicas, de un lado, avalan ciertos procesos sintácticos -lo cual constituye el objeto de interés de este trabajo-y, de otro, dan cuenta de la agramaticalidad de ciertos ejemplos. En este sentido, los ejemplos ad hoc de (7) muestran de forma sumaria los distintos tipos de reasunción nominal que los datos de (3) me han permitido abstraer:

(7) a. Tengo un libro que los dos primeros capítulos no tienen coherencia.

b. Conozco a una hija que la madre trabaja desde casa.

c. Conozco a un profesor que la letra no se entiende.

Estos ejemplos muestran que pueden operar como reasuntivos (i) los sustantivos de (7a), que contraen con el antecedente una relación de parte-todo; (ii) los sustantivos de (7b), los sustantivos propiamente relacionales, que, frente a los nombres 'predicativos', "are dependent on another referent in terms of how they themselves denote" (Pustejovsky, 1998: 18); y (iii) los sustantivos de (7c), que, frente a los de (7ab), no siempre habilitan la reasunción desde su estructura subléxica, pues la sintaxis fuerza a reinterpretar el SD reasuntivo como miembro de una relación de posesión en un sentido laxo, que incluye otros tipos de relaciones, como la de autor-obra. La hipótesis defendida aquí es que los SDs reasuntivos de (7a-b) muestran ciertas características en su estructura subléxica que habilitan la opción de que la sintaxis establezca una relación de ligamiento-A' entre el reasuntivo y el antecedente de la oración de relativo; dicha relación, sin embargo, redunda en agramaticalidad cuando la estructura subléxica no legitima la recategorización del SD reasuntivo como parte del antecedente (7a) o como relacional con respecto al antecedente (7b). No obstante, la reasunción no implica en ninguno de los dos casos un 'salto' o 'cambio' del tipo semántico, sea del antecedente, sea del SD reasuntivo.

En lo sucesivo, el artículo se estructura en una sección (apartado 3.1.), donde abordo los sustantivos de (7a) en una relación de parte-todo, alojada en su estructura subléxica, que no los faculta para intercambiar las posiciones sintácticas de antecedente y la que corresponda a la reasunción; la sección (apartado 3. 2) se consagra a las características del grupo de sustantivos relacionales de (7b), con unas características en su estructura subléxica que permiten que su argumento 'por defecto' (i. e. el que satura su quale formal), en términos de Pustejovsky (1998), intercambie libremente con el antecedente su posición sintáctica. Razones de espacio me han llevado a relegar los sustantivos de (7c) a investigaciones posteriores.

\section{Cuando las entradas léxicas codifican una información que la sintaxis habilita: Estructura subléxica y ligamiento- $\mathbf{A}^{\prime}$}

El objetivo de este apartado es explorar la posibilidad de que las entradas del léxico avalen, por la información codificada en su estructura subléxica, ciertos mecanismos 
sintácticos, como la reasunción nominal en cláusulas de relativo. En lo sucesivo, pretendo demostrar que la sintaxis de la reasunción exige que los SDs reasuntivos (a) estén categorizados como relacionales en su estructura subléxica, (b) se recategoricen como tales bajo ciertas condiciones (c) o se interpreten como miembros de una relación de parte-todo.

\section{1. Sustantivos en relación de parte-todo con el antecedente}

La explicación de los ejemplos de sustantivos en relación de parte-todo (7a) supra debe dar cuenta de las siguientes alternancias de gramaticalidad:

(8) a. Es un libro que el primer capítulo no tiene coherencia.

a'. *Es un capítulo que el libro no tiene coherencia.

b. Es un ordenador que el cargador no ha funcionado nunca.

b'. *Es un cargador que el ordenador no ha funcionado nunca.

c. Es una silla que las patas están torcidas.

c'. *Es una pata que la silla es muy incómoda.

d. Tengo una botella que el tapón no cierra bien.

d'. *Tengo un tapón que la botella es de un litro.

La generalización que permiten estos datos es que la posición de antecedente (i. e. núcleo [N, SN] o [Espec., SC], en el modelo de Kayne, 1994) no puede estar nunca saturada por el término que denota la parte en la relación de parte-todo ${ }^{6}$. Estos contrastes de gramaticalidad se extienden, curiosamente, a las construcciones con tópico vinculante de (9) y (10) (i. e. las Hanging Topic Left Dislocations de Cinque, 1990 y López, 2009, inter alia). Ciertas características dejan entrever la similitud entre este tipo de dislocaciones de la estructura informativa (Cinque, 1990; Zubizarreta, 1999; Zagona, 2002; Bosque \& Gutiérrez-Rexach, 2009) y las oraciones de relativo con pronombre reasuntivo; término este último que ya Cinque (1990) emplea para las construcciones con tópico vinculante. El pronombre reasuntivo de (9a) y (10a), que destaco, se opone al SD reasuntivo de (9b):

(9) a. [El ordenador $]_{j}$, sabes que $\mathbf{l o}_{\mathbf{j}}$ tienes que pagar a plazos.

b. $[\mathrm{El} \text { ordenador }]_{j}$, sabes que $[\text { el cargador }]_{j}$ no ha funcionado nunca.

c. $*[\text { El cargador }]_{\text {, }}$ sabes que [el ordenador $]_{j}$ no ha funcionado nunca.

(10) a. [La silla ], deberías haber sabido que tenías que cambiarla hace años.

b. [La silla $]_{j}$, deberías haber sabido que tenías que cambiar(le) [las patas $]_{j}$ hace años.

c. *[Las patas $]_{\mathrm{j}}$, deberías haber sabido que tenías que cambiar [la silla $]_{\mathrm{j}}$ hace años.

Las construcciones de tópico vinculante de (9) y (10) muestran que la reasunción pronominal de (9a) y (10a) puede involucrar también un SD, como en los ejemplos (b, c), coindizado con el sintagma en la posición de tópico superior, cabeza de la cadena 
de ligamiento-A'. En tanto que en la reasunción de (9) no media ninguna frontera sintáctica, las muestras de (10) ilustran cómo la reasunción asegura la gramaticalidad de la construcción a pesar de que la relación tópico-reasuntivo esté mediada por la frontera de Sintagma Complementante (SC), si bien tal gramaticalidad es tanto más dudosa cuanto mayor es el número de fronteras que median en la relación de ligamiento-A', asumidas las aportaciones de Chomsky (1986) y Boeckx (2012), inter alia. Retomando los ejemplos de (8), cuya explicación también es válida para los de (9) y (10), los rasgos subléxicos de los sustantivos permiten la relación de ligamiento-A' entre el reasuntivo y el sintagma que ocupa la posición de antecedente (i. e. [N, SN] o [Espec., SC]) de la oración de relativo en (8) y la posición de tópico superior en (9) y (10). En el modelo pustejovskyano del léxico, la interfaz léxico-sintaxis se concibe como una proyección desde la estructura de Qualia: cada quale legitima su proyección particular (Pustejovsky, 1998). Así, asumida esta concepción de la proyección de los rasgos subléxicos, la hipótesis sobre los ejemplos de (8) aquí defendida es que la relación de parte-todo que se establece entre reasuntivo y antecedente se explica por recurso al proceso de concordancia subléxica que media entre el quale constitutivo del reasuntivo y el quale constitutivo del antecedente. Los dos ítems léxicos deben, pues, concordar en el quale constitutivo; en el caso de que no se verifique tal concordancia, la expresión deriva en agramaticalidad. Los ejemplos de (11) ilustran que los SDs reasuntivos no pueden interpretarse como partes del antecedente de la cláusula de relativo:

(11) a. *Es [un ordenador] j que [las patas] j son demasiado débiles.

b. *Es [un libro]j que [el tapón] j no cierra bien.

c. ${ }^{*}$ Es [un enchufe $]_{j}$ que $[\text { la mesa }]_{j}$ no ha funcionado nunca.

Para ilustrar este razonamiento, por el que la estructura subléxica debe codificar la relación de parte-todo del sustantivo, recojo en (12) los dos niveles de representación, pertinentes al caso, que subyacen al ítem 'capítulo' (i. e. ligado-A') del ejemplo (8a-a'), y en (13) muestro el análisis equivalente para el ítem 'libro’ (i. e. antecedente de la cláusula):

(12)

$$
\left[\begin{array}{l}
\text { ARGSTR }=\left[\begin{array}{l}
\text { ARG1 }=\text { x:información } \\
\text { ARG2 }=\text { y:obj_fí́sico }
\end{array}\right] \\
\text { QUALIA }=\left[\begin{array}{l}
\text { información·obj_físico_lcp } \\
\text { formal }=\text { contener }(\mathrm{y}, \mathrm{x}) \\
\text { télico }=\text { procesar }(\mathrm{e}, \mathrm{z}, \mathrm{x}) \\
\text { agentivo }=\text { escribir }(\mathrm{e}, \mathrm{z}, \mathrm{x}) \\
\text { constitutivo }=\text { parte_de(x,w:libro })
\end{array}\right]
\end{array}\right.
$$


(13)

$\left[\begin{array}{l}\text { libro } \\ \text { ARGSTR }=\left[\begin{array}{l}\text { ARG1 }=\text { x:información } \\ \text { ARG2 }=\text { y:obj_físico }\end{array}\right] \\ \text { QUALIA }=\left[\begin{array}{l}\text { información·obj_físico_lcp } \\ \text { formal }=\text { contener }(y, x) \\ \text { télico }=\text { leer }(\mathrm{e}, \mathrm{w}, \mathrm{y} \cdot \mathrm{x}) \\ \text { agentivo }=\text { escribir(e,w,x.y) } \\ \text { constitutivo }=\text { tiene_como_parte }\end{array}\right] \\ \text { (x,z:capítulo) }\end{array}\right]$

El sistema de tipos de (12) y (13) evidencia que a los ítems léxicos subyacen dos niveles de representación: la estructura argumental y la estructura de Qualia (Pustejovsky, 1991, 1998), de la que parte la proyección de los rasgos subléxicos en la sintaxis y en la que se especifica (a) el quale formal, que, en los tipos simples, coincide con el tipo del argumento; (b) el quale télico, que hospeda la información relativa a aquello para lo que está destinado; (c) el quale agentivo, que recoge el evento en virtud del cual se crea el ítem léxico y, por último, (d) el quale constitutivo, donde se especifica de qué manera se relaciona el ítem léxico con las partes que lo componen y define "what that object is logically part of, if such relation exists" (Pustejovsky, 1998: 98). Es en el quale constitutivo de 'capítulo' (12) donde he albergado una instrucción subléxica, parte_de (ing. part_of), que define el ítem en términos de las relaciones lógicas que contrae, en este caso, con los conjuntos de los que forma parte. Cada sistema de tipos muestra una estructura de Qualia encabezada por información·obj_físico_lcp, por tratarse de 'paradigmas léxico-conceptuales' (Pustejovsky \& Anick, 1988; Ježek \& Melloni, 2011; De Miguel \& Batiukova, 2017 para la 'copredicación') que pueden aludir tanto al soporte como a la información que este contiene.

Presente ya en el trabajo de Busa, Calzolari, Lenci y Pustejovsky (2001), la instrucción has_as_part, que traduzco como tiene_como_parte, en el quale constitutivo del ítem léxico 'libro', proviene de la estructura de qualia extendida, desarrollada, entre otros lugares, en Ruimy (2006a, 2006b, 2006c) y Monachini, Quochi, Ruimy y Calzolari (2007), donde se amplía cada uno de los cuatro componentes de la estructura de Qualia originaria (Pustejovsky, 1991, 1998). Por lo que respecta al ejemplo (13), la instrucción has_as_part, similar a otras como made_of o has_as_member, codifica la relación que guarda el ítem 'libro' con respecto a 'capítulo', así como con respecto a 'página', 'sección', 'epígrafe', etc., todos ellos reasuntivos potenciales de oraciones de relativo encabezadas por 'libro', como ilustran someramente los ejemplos de (14):

(14) a. Es un libro que las primeras páginas son ilegibles.

b. Es un libro que las dos primeras secciones no tienen coherencia.

c. Es un libro que los epígrafes no tienen sentido? 
La Figura 1 ilustra cómo debe verificarse la relación de ligamiento entre los dos quales constitutivos, para lo que hago uso de la línea discontinua -redundante por estar la relación entre los qualia expresa a través del sistema de variables-. Si bien empleo el par 'libro' / 'capítulo' por coherencia expositiva, sirvan a este respecto otros pares como los recogidos en (8), como 'ordenador' / 'cargador', 'silla' / 'patas', etc.:

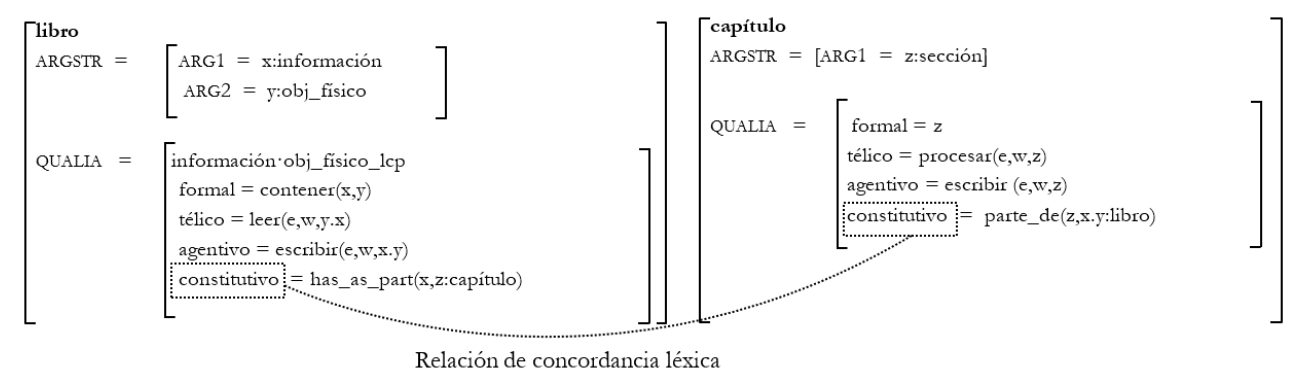

Figura 1. Relación de concordancia léxica: Quale constitutivo.

El sistema de variables de las representaciones léxico-semánticas de (12) y (13) ha sido modificado con la finalidad de dar cuenta, por un lado, de la correferencia necesaria entre los valores correspondientes a 'capítulo', para el que he adoptado el valor $\mathrm{z}$; y, por otro lado, de la correferencia posible, no necesaria, del agente (valor w) de los eventos albergados en los valores télico, formal y agentivo, en los que la variable e no indica que el ítem léxico denote por sí un evento, sino que su significado está íntimamente relacionado con ciertas actividades, como la lectura o la escritura (Pustejovsky, 2013; De Miguel \& Batiukova, 2017). Asumida, pues, la inclusión de la variable e en la representación léxico-semántica, la reasunción se revela habilitada desde la información subléxica: si un elemento, a pesar de definirse por constituir la parte de un todo, no está vinculado por medio de su quale constitutivo con el antecedente de la cláusula de relativo en la que aparece, la construcción no cumple las condiciones de buena formación y redunda, por ello, en agramaticalidad, como se prueba en (15), donde la coindización disjunta implica referencia disjunta:

(15) a. Es [un libro]; que [el primer capítulo]; no tiene coherencia.

b. *Es [un libro]; que [el primer capítulo] ${ }_{x}$ no tiene coherencia.

c. ${ }^{*}$ Es [un libro]; que [el primer capítulo de tu libro] $]_{\mathrm{x}}$ no tiene coherencia.

Frente a (15a), que satisface las condiciones de buena formación, (15b) y (15c) las infringen por no estar reasuntivo y antecedente en una relación de parte-todo, que aquí he señalado con subíndices; en otros términos, dado que no se verifica una concordancia entre los dos quales constitutivos, la construcción está excluida de la gramática. Así, la muestra de (15a), junto a las oraciones bien formadas de (8), evidencia un fenómeno de concordancia 'plena' o 'selección semántica' (Pustejovsky, 1998) de rasgos subléxicos: los qualia constitutivos de cada uno de los términos involucrados en la relación de concordancia subléxica parecen avenirse sin necesidad de recurrir a ningún mecanismo generativo adicional. En este sentido, la reasunción 
no altera el tipo semántico del sustantivo que ocupa la posición de núcleo del SN en el interior del SD reasuntivo, sino que establece una concordancia entre los qualia ya especificados en su representación léxico-semántica.

El rasgo distintivo de los $\mathrm{SDs}$ reasuntivos en relación de parte-todo con el antecedente es que están constituidos por nombres predicativos, frente a los sustantivos relacionales que abordaré en la sección (apartado 3.2.), cuya referencia necesita de un argumento, sintácticamente implícito o explícito, que los sature.

\subsection{Sustantivos relacionales en función reasuntiva}

La bibliografía consagrada al estudio de los nombres relacionales conviene en la naturaleza 'dependiente' de esta forma de referencia nominal; en rigor, en términos de Pustejovsky (1998: 151) "a relational noun denotes a set of individuals standing in relation to at least one other individual in a specific way", dependencia asimismo recogida en De Bruin y Scha (1988). El rasgo que distingue estos sustantivos de los propiamente predicativos es que no están semánticamente saturados, por lo que, en el plano semántico, los nombres relacionales se conciben como funciones que deben aplicarse a un argumento (De Bruin \& Sha, 1988; Ingria \& George, 1993), denominado ‘correlato' en Lander (2000), 'argumento interno' en Eschenbach (1993) y 'argumento por defecto' en Pustejovsky (1998).

Los sustantivos relacionales conforman un grupo más o menos heterogéneo heterogeneidad que, en rigor, tiene repercusiones para la gramática (Eschenbach, 19938; Lander, 2000)-: sustantivos de parentesco ('padre', 'tía', 'nieta') y sustantivos de función (De Bruin \& Sha, 1988), como 'distancia', 'anchura', 'velocidad', etc. que, cuando se aplican a un argumento, devuelven la denotación precisa de un valor numérico. Tanto los sustantivos de parentesco como los de función se conciben como dependientes con respecto a otro sustantivo, que puede aparecer en su mismo sintagma, en la oración o en el contexto discursivo previo.

La incardinación en la teoría lingüística de la dependencia referencial de estos sustantivos ha sido fuente de controversia. Ciertas aportaciones primigenias, como De Bruin y Scha (1988), Stallard (1989) e Ingria y George (1993), se sirvieron de distintos mecanismos teóricos para formular el carácter relacional de algunos sustantivos: la similitud que guardan tales propuestas es el recurso al argumento adicional del que el sustantivo relacional obtiene la referencia. A Pustejovsky y Anick (1988), sin embargo, se debe la formulación de que el carácter relacional y, en definitiva, el argumento del sustantivo relacional han de incardinarse en la estructura del ítem léxico:

"If the denotation of one sense of a lexical item is "dependent" on another, then that dependency is part of the semantic representation of that lexical item. A familiar example is father or mother, where the relational nature must be part of the semantics of the noun" (Pustejovsky \& Anick, 1988: 519). 
Este tipo de nombres recibe aquí la denominación de 'nombres relacionales primitivos', término que traduzco del original (ing. primitive relational nominals). En Pustejovsky (1998: 151), si bien se abandona el término, se establece que "a relational noun denotes a set of individuals standing in relation to at least one other individual in a specific way". De ello se desprende una estructura como la de (16), tomada de Pustejovsky (1998: 152):

$$
(16)\left[\begin{array}{l}
\text { brother } \\
\text { ARGSTR }=\left[\begin{array}{l}
\text { ARG1 }=\text { x:human } \\
\text { D-ARG1 =y:human }
\end{array}\right] \\
\text { QUALIA }=\left[\begin{array}{l}
\text { formal }=\text { male }(\mathrm{x}) \\
\text { const }=\text { brother_of }(\mathrm{x}, \mathrm{y})
\end{array}\right]
\end{array}\right]
$$

La representación léxico-conceptual de (16) recoge, como parte del quale constitutivo del sustantivo relacional brother, la dependencia referencial por medio de la instrucción brother_of 'hermano de', que remite al argumento que satura la posición asignada (identificada con y) y que constituye un argumento por defecto (ingl. default argument), denominado argumento 'interno' por Eschenbach (1993). Pustejovsky (1998: 63) define esta clase de argumentos 'por defecto' como: "Parameters which participate in the logical expressions in the qualia, but which are not necessarily expressed syntactically".

Asumido, pues, el carácter subléxico de la dependencia referencial de este tipo de sustantivos, examinaré los datos recogidos en (17) y (18):

(17) a. Conozco a una hija que la madre trabaja desde casa.

b. Conozco a una madre que la hija trabaja desde casa.

c. Es un hijo que los padres no han trabajado nunca.

d. Son unos padres que el hijo no ha ido nunca a la escuela.

e. *Son unos padres que tu hijo no ha ido nunca a la escuela.

(18) a. Tengo una abuela que la tía trabaja en El País.

b. Tengo una tía que la abuela trabaja en El País.

c. Conozco a una amiga que el sobrino es tenor.

d. Tengo una prima que la abuela por parte de madre trabaja en El País.

c. *Tengo una sobrina que mi tía trabaja desde casa.

La distinción entre (17) y (18) no es gratuita. Todos los ejemplos planteados comparten el carácter referencial de, al menos, el sustantivo en función reasuntiva ubicado en el interior de la oración de relativo. Los ejemplos de (17) se diferencian de los de (18) en que el sustantivo que opera como antecedente y el que figura en el interior de la cláusula como reasuntivo saturan mutuamente su contenido subléxico; en otros términos, antecedente y reasuntivo obtienen su referencia en virtud de la 
presencia de su respectivo argumento por defecto (y en la representación léxicoconceptual de la Figura 2 infra) bien en la posición del antecedente, bien en la posición sintáctica que corresponda a la función reasuntiva. La concordancia del quale formal, por tanto, es plena, como recojo en la Figura 2:

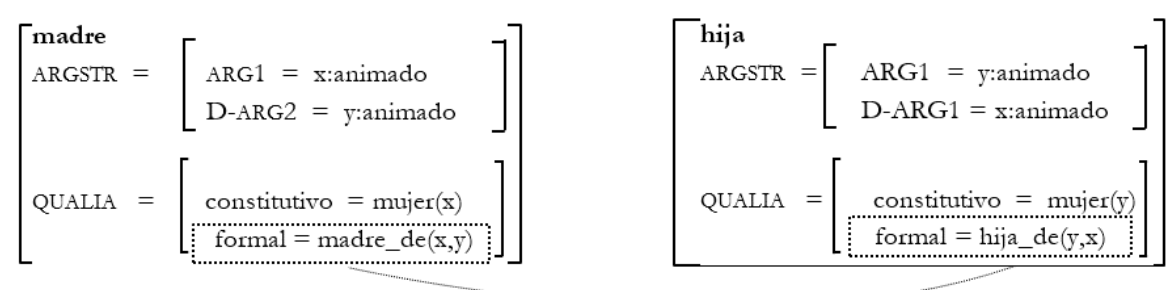

Figura 2. Relación de concordancia léxica: Quale. formal

Como en la Figura 1 supra, me sirvo en la Figura 2 del símbolo gráfico redundante que señala la concordancia en los quales formales de 'madre' e 'hija' que corresponden al ejemplo (17b). En rigor, el sistema de variables indica por sí mismo que el argumento $x$, que corresponde al sustantivo 'madre', tiene $y$ como argumento por defecto, que corresponde a 'hija'. La relación de parentesco está, así, integrada en la representación léxico-semántica del sustantivo relacional, que ve su argumento por defecto saturado en virtud de la reasunción.

La reasunción no siempre ocasiona que antecedente y reasuntivo saturen mutuamente la posición del argumento por defecto. Esta aserción perfila la diferencia de los ejemplos del grupo (18) con respecto a los anteriores: los sustantivos relacionales en función reasuntiva no siempre obtienen su referencia plena del sustantivo en la posición sintáctica del antecedente, pues 'abuela' no se define por recurso a 'tía' ni 'sobrino' se define por recurso a 'amiga'. Sin embargo, tales suposiciones precisan una matización: los sustantivos relacionales en función reasuntiva, en la misma línea que los sustantivos relacionales en oraciones simples, pueden aparecer desprovistos del argumento que los satura. Esta es, de hecho, una intuición percibida en De Bruin y Scha (1988), en Ingria y George (1993) y en Pustejovsky (1998), cuya solución teórica para los nombres relacionales, aducida en (16) supra, da cuenta de esta posibilidad asumiendo que el argumento es D-ARG 'argumento por defecto', que puede así aparecer explícita o implícitamente. Si este argumento por defecto muestra una variable libre, el sustantivo relacional no está saturado.

La sintaxis del español permite ilustrar nítidamente este razonamiento: en una oración como "Los padres llegaron más temprano de lo que se pensaba", aunque el argumento 'hijo/a/s' no figure explícitamente, el sustantivo 'padres' puede interpretarse en función del discurso previo o posterior. La misma situación representan las oraciones de carácter genérico ('Los padres tienen que saber qué hacer en todo momento'), lo que prueba que, en consonancia con Pustejovsky (1998), el 
argumento de los nombres relacionales es 'por defecto', carácter facultativo asimismo señalado en De Bruin y Scha (1988), Eschenbach (1993) y Lander (2000). Este razonamiento, sin embargo, no debe esbozarse con independencia de qué posición sintáctica ocupa el sustantivo relacional en cuestión. Obsérvense, a este respecto, los ejemplos de (19):

(19) a. Conozco a una amiga que el sobrino es tenor.

a'. ??/*Conozco (a) un sobrino que la amiga es soprano.

b. Tengo un sobrino que la amiga es soprano.

b’. Tengo una amiga que el sobrino es tenor.

Las muestras de (19) revelan datos interesantes sobre los sustantivos relacionales y, en rigor, permiten postular la trascendencia a la sintaxis de instrucciones de naturaleza subléxica. La oración de (19a') muestra un sustantivo relacional '(un) sobrino' que no ve saturada la posición del argumento por defecto de su estructura subléxica, pues el reasuntivo '(la) amiga' no puede formar parte de su quale formal, lo cual redunda en la mala formación (o 'extrañeza') de la expresión en cuestión. Sin embargo, la buena formación de la construcción de (19a) se debe a que el sustantivo relacional '(el) sobrino' se interpreta como sobrino_de (x,y), siendo (x) ‘el sobrino' e (y) 'una amiga'. Sirva la Figura 3 para ejemplificar este razonamiento:

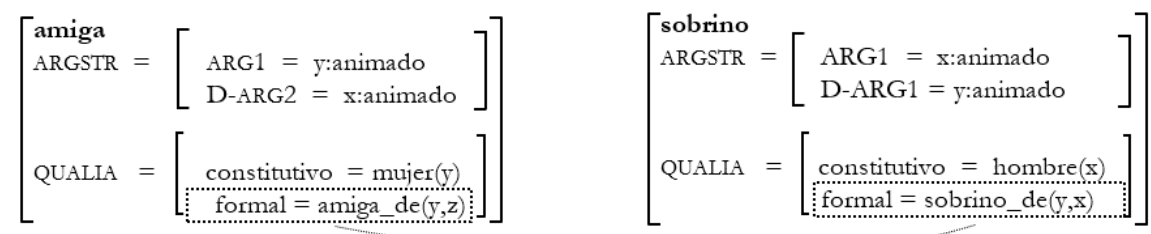

Figura 3. Relación de concordancia léxica: Quale formal.

Siguiendo la terminología de Eschenbach (1993), la voz 'amigo' se define por una relación simétrica, en tanto que 'sobrino' representa una constelación asimétrica. Las dos voces guardan, en la oración propuesta, una cierta dependencia referencial. A pesar de la similitud entre la figura 2 y la 3, un matiz necesario permite diferenciarlas: la variable z alojada en el quale formal del ítem 'amiga' refleja que, en este caso, 'sobrino' no forma parte de su estructura subléxica. Aunque el SD 'el sobrino', en posición de [Especificador, SFlex] y de carácter reasuntivo, se define por recurso al SD 'la amiga', que ocupa la posición de antecedente de la cláusula de relativo (i. e. [N, $\mathrm{SN}$ ] o [Especificador, SC], según el modelo), el quale formal de este último ítem es saturado por la variable $z$, que, en el caso particular de (19a), se corresponde con el elemento pronominal 'pro' asociado a la flexión de una forma verbal como 'conozco'. En definitiva, la diferencia entre la figura 2 y la 3radica en que no existe 'reciprocidad de saturación' en esta última, en la medida en que los sustantivos relacionales en cuestión no son argumentos por defecto el uno del otro. La relación entre 'amiga' y 
'sobrino' de (19a) es, por ende, asimétrica, frente a la simetría en la saturación de los ejemplos (17a-d), esquematizada en la Figura 2.

Por su parte, los datos del par (19b-b') muestran una diferencia clara con respecto a (19a-a'): el verbo 'conocer' no impone a su complemento una relación de parentesco que el verbo 'tener' sí implica, por tratarse de uno de los relation-denoting lexical element 'elementos léxicos de relación' a los que aluden De Bruin y Scha (1988), algunas de cuyas propiedades son objeto de estudio en Ingria y George (1993). Con respecto a esta clase de elementos, se ha defendido que:

"Relational nouns can be connected to their arguments/values by a variety of verbs and prepositions, which constitute a semantic complex that is also used, with exactly the same structure but with a different meaning, to operate on non-relational nouns [...]. These terms (like "of", "bave" and "with") are highly polysemous, and any language processing system must encompass mechanisms for disambiguating their intended meaning in any particular utterance" (De Bruin \& Scha, 1988: 26-27).

Se trata, por tanto, de mecanismos de los que se sirven las lenguas para vincular un nombre relacional con los argumentos que lo saturan. Así, 'tener' en los ejemplos (19b-b') conecta '(un) sobrino' con el elemento pronominal 'pro' asociado a la Flexión de la forma verbal 'tengo'. Por añadidura, cualquier otra interpretación de (19b-b') en la que 'un sobrino' o 'una sobrina' no esté vinculado con el hablante se descarta por agramatical, como prueban los ejemplos que recojo en (20), donde (20d) muestra que el verbo existencial 'haber', frente a 'tener', no es uno de los elementos léxicos de relación de De Bruin y Scha (1988), porque no puede asignar al sustantivo relacional un argumento que lo sature:

(20) a. (pro); Tengo un sobrinoj que el amigo es contratenor.

b. (pro) i Tengo un sobrino $\left({ }^{*}\right.$ tuyo $\left._{\mathbf{x}}\right)$ que el amigo es contratenor.

c. (pro); Tienes un sobrino $\left({ }^{*}\right.$ míox $\left._{\mathrm{x}}\right)$ que el amigo es contratenor.

d. *Hay un sobrino que la amiga es soprano.

e. ?? (pro) Tengo un hermano que la hermana trabaja desde casa.

Estos contrastes de agramaticalidad se extienden al SD reasuntivo, como se detalló con respecto a los ejemplos (15) supra. Así, los casos de referencia disjunta -en un sentido lato de 'referencia'- entre el reasuntivo y el antecedente de la oración de relativo redundan en agramaticalidad. La extrañeza del ejemplo (20e), que debo al comentario de un revisor anónimo, se deriva del hecho de que el sustantivo relacional simétrico 'hermano', antecedente de la oración de relativo, sea saturado por el 'pro' relacionado con la Flexión, lo cual parece constreñir la posibilidad de que sea argumento por defecto del SD en función reasuntiva9. En rigor, estos datos de la reasunción deben parangonarse con los que Staroverov (2007) aduce en relación con la denominada 'conjunción recíproca', donde cada uno de los términos es relacional con respecto al otro, como en (24), 
(21) La novela es sobre un marido y una mujer. [ejemplo adaptado de Staroverov, 2007: 303]

donde los dos nombres relacionales aluden a dos personas que son 'marido' y 'mujer' el uno del otro; cualquier otra lectura, de hecho, se concibe como excluida de la gramática. En cualquier caso, cotejados con los ejemplos de sustantivos en relación de parte-todo de (8), que derivan en agramaticalidad /extrañeza si hay inversión, los datos de (17-18) dejan entrever que las instrucciones codificadas en el léxico habilitan ambas posiciones en la estructura sintáctica para cada uno de los términos de la relación de dependencia referencial. Esta apreciación permite intuir, en cierta medida, que la sintaxis de la reasunción debe acceder al contenido que las palabras hospedan en su estructura subléxica, cuyas instrucciones legitiman los procesos de ligamiento- $\mathrm{A}$ ' que exige la reasunción.

En este sentido, el proceso que opera en la interfaz léxico-sintaxis parece ser el mismo que para los ejemplos de relación parte-todo de (8)-(15): se verifica una concordancia de rasgos subléxicos a distancia, pues reasuntivo y antecedente deben codificar en el interior de su estructura de Qualia un quale constitutivo, en el caso de los reasuntivos en relación de parte-todo, o un quale formal, en el caso de los sustantivos relacionales, que explicite la mutua dependencia referencial. Tal solución teórica, sin embargo, reviste un valor indudablemente hipotético y se halla, así, sujeta a revisión.

\section{Recapitulación, conclusiones y posibles vías de indagación}

En el desarrollo de este trabajo, después de una breve introducción al concepto de reasunción y una primera aproximación a la documentación del fenómeno, he intentado demostrar por medio de ejemplos y contraejemplos la necesidad de defender que los rasgos del léxico se proyectan en la sintaxis; en otras palabras, los rasgos subléxicos habilitan el funcionamiento de ciertos mecanismos sintácticos $\mathrm{y}$, en cierta medida, los legitiman. De ello se deriva, a modo de corolario, que dos sustantivos no concordados en términos subléxicos, uno en la posición de antecedente y otro en el interior de la cláusula de relativo, no podrán establecer entre sí una relación de ligamiento-A' y garantizar, con ello, la buena formación de la construcción reasuntiva. Con ello, este estudio de la reasunción en términos subléxicos pretende enriquecer la descripción de las 'estructuras del léxico' (De Miguel, 2006) y de los mecanismos por los que estas estructuras se proyectan en la sintaxis.

No debe, sin embargo, soslayarse el hecho de que la sintaxis parece imponer también ciertos requisitos que, de hecho, tienen relevancia distribucional en el sustantivo. En rigor, la imposibilidad de que un constituyente nominal reasuntivo figure sin determinante da cuenta de la agramaticalidad de ejemplos como *"Soy una 
persona que intereses son muchos" o *'Tienes un ordenador que cargadores no funcionan", en la medida en que la reasunción se ratifica por medio del determinante. En investigaciones posteriores, el sistema de representaciones subléxicas de sustantivos en función reasuntiva habrá de relacionarse con la estructura articulada del sintagma determinante en español. Dado que es también en la sintaxis donde se establecen las relaciones de ligamiento-A', resta aún por determinar cuál es la coda de la cadena: los elementos pronominales, por ejemplo, una vez ligados, abandonan su significado variable para adoptar un valor constante, si bien no es este el caso de los nominales. Aunque existen ciertas propuestas de 'nombres ligados', que he encontrado en Asudeh (2005) y Borer (2013), entre otros, la condición de que una expresión$\mathrm{R}$ (eferencial) esté bajo ligamiento contraviene uno de los presupuestos básicos de la teoría del ligamiento de Chomsky (1980) y Chomsky (1981). No obstante, la solución teórica pudiera estar en considerar que, antes de que se establezca una relación de ligamiento-A', los sustantivos aquí sometidos a estudio no son expresiones$\mathrm{R}$ (eferenciales) strictu sensu: su valor referencial vendría determinado por el sistema de variables que antecedente y reasuntivo comparten, como se ha ilustrado en las secciones previas.

Por estas y otras razones, creo que los datos discutidos a lo largo del artículo me facultan para abrir una modesta vía de estudio: la postulación de variables en los qualia que expliquen cómo las relaciones de ligamiento- $\mathrm{A}^{\prime}$ acceden al interior de la estructura de las palabras y seleccionan, previa legitimación por parte del léxico, cuáles de los rasgos se deben someter a ligamiento.

\section{REFERENCIAS BIBLIOGRÁFICAS}

Andrews, A. D. (2007). Relative clauses. En T. Shopen (Ed.), Language Typology and Syntactic Description. Volume II: Complex Constructions (pp. 206-236). Cambridge: Cambridge University Press.

Anick, P. \& Bergler, S. (1991). Lexical structures for linguistic inference. En J.Pustejovsky \& S. Bergler (Eds.), Lexical Semantics and Knowledge Representation. Proceedings of the First SIGLEX Workshop (pp. 121-135). Berlín / Heidelberg: Springer-Verlag.

Asudeh, A. (2005). Relational nouns, pronouns and resumption. Linguistics and Philosophy, 28, 375-446.

Bentivoglio, P. (2003). Las construcciones de retoma en las cláusulas relativas: Un análisis variacionista. En F. Moreno Fernández, F. Giménez Menéndez, J. A. Samper, Ma L. Gutiérrez Araus, M. Vaquero \& C. Hernández (Coords.), Lengua, variación y contexto. Estudios dedicados a Humberto López. Morales (pp. 507520). Madrid: Arco Libros. 
Boeckx, C. (2012). Syntactic islands. Cambridge: Cambridge University Press.

Brucart, J. M.(1999). La estructura del sintagma nominal: Las oraciones de relativo. En I. Bosque \& V. Demonte (Dirs.), Gramática descriptiva de la lengua española (pp. 395-522). Madrid: Espasa-Calpe.

Borer, H. (2013). Taking form. Structuring sense Volume III. Oxford: Oxford University Press.

Bosque, I. \& Gutiérrez-Rexach, J. (2009). Fundamentos de sintaxis formal. Madrid: Akal.

Busa, F., Calzolari, N., Lenci, A. \& Pustejovsky, J. (2001). Building a semantic lexicon: Structuring and generating concepts. En H. Bunt, R. Muskens \& E. Thijsse (Eds.), Computing Meaning (pp. 29-51). Dordretcht: Kluwer Academic Publishers.

Caviglia, S. \& Malcuori, M. (2007). Las oraciones de relativo en la interfaz gramáticadiscurso. En V. Bertolotti, S. Caviglia, S. Costa, M. Grassi, M. Malcuori \& M $^{\mathrm{a}}$ D. Muñoz Núñez (Eds.), Estudios de lingüística hispánica (pp.43-69). Cádiz: Servicio Publicaciones de la Universidad de Cádiz.

Cerrón-Palomino, Á. (2006). Pronombres de retoma en cláusulas relativas del castellano peruano: Un fenómeno de causación múltiple. Lexis, 30(2), 231258 .

Chomsky, N. (1980). On binding. Linguistic Inquiry, 11(1), 1-46.

Chomsky, N. (1981). Lectures on government and binding. The Pisa lectures. Dordretch: Foris.

Chomsky, N. (1986). Barriers. Massachusetts: The MIT Press.

Cinque, G. (1990). Types of $\bar{A}$-dependencies. Massachusetts: The MIT Press.

Comrie, B. (1981). Language Universals and Linguistic Typology. Oxford: Blackwell.

Comrie, B. (2004). Tipología sintáctica: ¿Hasta qué punto son exóticas las oraciones de relativo de las lenguas de tipo europeo? En J. Gil \& R. Mairal (Eds.), En torno a los universales lingüisticos (pp. 171-198). Madrid: Akal.

Comrie, B. \& Keenan, E. (1979). Noun phrase accessibility revisited. Language, 55(3), 649-664.

Cristofaro, S. \& Giacalone Ramat, A. (2007). Relativization patterns in the languages of Europe. En P. Ramat \& E. Roma (Eds.), Europe and the Mediterranean as Linguistic Areas. Convergencies from a historical and typological perspective (pp. 63-93). Amsterdam / Philadelphia: John Benjamins. 
De Benito Moreno, C., Pueyo, J. \& Fernández-Ordóñez, I. (2016). Creating and designing a corpus of rural Spanish. En D. Misra Sharma, R. Sangal \& A. Kumar Singh (Eds.), Procceedings of the 13th Conference on Natural Language Processing (KONVENS) (pp. 78-83). Varanasi: NLP Association of India.

De Bruin, J. \& Sha, R. (1988). The interpretation of relational nouns. En el 26th Annual Meeting of the Association for Computational Linguistics: Proceedings of the Conference (pp. 25-32). Morristown: Association of Computational Linguistics.

De Miguel, E. (2006). Cosas que ocurren dentro de las palabras y entre palabras: Datos e hipótesis acerca de la estructura del léxico y de ciertas estructuras léxicas. En E. De Miguel, A. Palacios \& A. Serradilla (Eds.), Estructuras Léxicas y Estructura del Léxico (pp. 7-31). Frankfurt: Peter Lang.

De Miguel, E. (2009). La teoría del lexicón generativo. En E. De Miguel (Ed.), Panorama de la lexicología (pp. 337-370). Barcelona: Ariel.

De Miguel, E. (2012). Properties and internal structure of the lexicon: Applying the Generative Lexicon Model to Spanish. En M. Sanz \& J. M. Igoa (Eds.), Applying Language Science to Language Pedagogy: Contributions of Linguistics and Psycholinguistics to Second Language Teaching (pp. 165-200). Newcastle Upon Tyne: Cambridge Scholars Publishing.

De Miguel, E. (2014). La lexicología en España. Tendencias y proyectos en curso. En R. García Pérez (Dir.), La lexicologie en Espagne: De la lexicologie a la lexicographie (pp. 17-44). París: Cahiers de lexicologie.

De Miguel, E. \& Batiukova, O. (2017). Compositional mechanisms in a generative model of the lexicon. En S. Torner \& E. Bernal (Eds.), Collocations and Other Lexical Combinations in Spanish. Theoretical, lexicographical and applied perspectives (pp. 92-113). Londres/Nueva York: Routledge.

Engdahl, E. (1985). Parasitic gaps, resumptive pronouns, and subject extractions. Linguistics, 23(1), 3-44.

Eschenbach, C. (1993). Semantics of number. Journal of Semantics, 10(1), 1-31.

Fernández-Ordóñez, I. (Dir.) (2005). Corpus Oral y Sonoro del Español Rural [en línea]. Disponible en: www.corpusrural.es ISBN 978-84-616-4937-2.

Fernández-Ordóñez, I. (2007). Nuevas perspectivas en el estudio de la variación dialectal del español: El Corpus Oral y Sonoro del Español Rural (COSER). En D. Trotter (Ed.), Actes du XXIV Congrès de Linguistique et Philologie Romanes (Aberystwyth, Wales, 2-5 August 2004) (pp. 29-44) vol. 3. Tübingen: Niemeyer. 
Fernández-Ordóñez, I. (2009). Dialect grammar of Spanish from the perspective of the Audible Corpus of Spoken Rural Spanish (or Corpus Oral y Sonoro del Español Rural, COSER). Dialectologia, 3, 23-51.

Fernández-Ordóñez, I. (2010). New methods for the study of grammatical variation and the Audible Corpus of Spoken Rural Spanish. En G. Aurrekoetxea \& J. L. Ormaetxea (Eds.), Tools for Linguistic Variation (pp. 119-30). Bilbao: Universidad del País Vasco.

Fernández-Ordóñez, I. (2011). Nuevos horizontes en el estudio de la variación gramatical del español: El Corpus Oral y Sonoro del Español Rural. En G. Colón Domènech \& Ll. Gimeno Betí (Eds.), Noves tendències en la dialectología contempoània (pp. 173-203). Castelló de la Plana: Universitat Jaume I.

Givón, T. (1975). Promotion, NP accessibility, and case marking: T understanding grammars. Working Papers on Language Universals, Universidad de Stanford, USA.

Givón, T. (1979). On understanding grammar. Nueva York/San Francisco/Londres: Academic Press.

Givón, T. (2001). Syntax. An introduction. Amsterdam/Philadelphia: John Benjamins.

Heylen, D. (1995). Lexical functions, generative lexicons and the world. En P. SaintDizier \& E. Viegas (Eds.). Computational lexical semantics (pp. 125-140). Cambridge: Cambridge University Press.

Ingria, R. J. P \& George, L. M. (1993). Adjectives, nominals, and the status of arguments. En J. Pustejovsky (Ed.), Semantics and the Lexicon (pp. 107-127). Dordrecht/Boston/Londres: Kluwer Academic Publishers.

Ježek, E. \& Melloni, Ch. (2011). Nominals, polysemy, and co-predication. Journal of Cognitive Science, 12, 1-31.

Kayne, R. (1994). The antisymmetry of syntax. Massachusetts: The MIT Press.

Keenan, E. L. (1972). On semantically based grammar. Linguistic Inquiry, 3(4), 413-461.

Keenan, E. L. (1985). Relative clauses. En T. Shopen (Ed.), Language typology and syntactic description (pp. 141-170). Cambridge: Cambridge University Press.

Keenan, E. L. \& Comrie, B. (1977). Noun phrase accessibility and universal grammar. Linguistic Inquiry, 8(1), 63-99.

Keenan, E. L. \& Comrie, B. (1979). Data on the noun phrase accessibility hierarchy. Linguistic Inquiry, 55(2), 333-351. 
Kroch, A. (1981). On the role of resumptive pronouns in amnestying island violations. En R. Hendrick, C. Masek \& M. F. Miller (Eds.), Papers from the Seventeenth Regional Meeting of the Chicago Linguistic Society (pp. 125-135). Chicago: Chicago Linguistic Society.

Lander, Y. (2000). K типологии релячионных имен". ms. Universidad Estatal de Rusia para las Humanidades [en línea]. Disponible en: https://www.academia.edu/1702134/К_типологии_реляционных_имен

López, L. (2009). A derivational syntax for information structure. Oxford: Oxford University Press.

Lope Blanch, J. M. (1986). Estudios de lingüistica española. México: Universidad Nacional Autónoma de México.

Lope Blanch, J. M. (2001). Los relativos en problemas. Lexis, 25, 159-172.

Maxwell, D. (1979). Strategies of relativization and NP accessibility. Language, 55(2), 352-371.

McCloskey, J. \& Hale, K. (1984). On the syntax of person-number inflection in modern Irish. Natural Language and Linguistic Theory, 1(4), 487-533.

Monachini, M., Quochi, V., Ruimy, N. \& Calzolari, N. (2007). Lexical relations and domain knowledge: The biolexicon meets the qualia structure, ms. Ponencia presentada en GL2007: 4th International Workshop on Generative Approaches to the Lexicon [en línea]. Disponible en: https://www.researchgate.net/profile/Valeria_Quochi/publication/2517788 61_Lexical_Relations_and_Domain_Knowledge_The_Bio-

Lexicon_Meets_the_Qualia_Structure/links/58529ba708aef7d030a51042/Le xical-Relations-and-Domain-Knowledge-The-Bio-Lexicon-Meets-the-QualiaStructure.pdf

Piera, C. (2009). Una idea de la palabra. En E. De Miguel (Ed.), Panorama de la lexicología (pp. 25-49), Barcelona: Ariel.

Prince, E. F. (1990). Syntax and discourse: A look at resumptive pronouns. En D. J. Costa (Ed.), Proceedings of the Sixteenth Annual Meeting of the Berkeley Linguistics Society (pp. 482-497). California: Berkeley Linguistic Society.

Pustejovsky, J. (1991). The generative lexicon. Computational Linguistics, 17(4), 409-441.

Pustejovsky, J. (1995). Linguistic constraints on type coercion. En P. Saint-Dizier \& E. Viegas (Eds.), Computational Lexical Semantics (pp. 71-97). Cambridge: Cambridge University Press.

Pustejovsky, J. (1998). The generative lexicon. Massachusetts: The MIT Press. 
Pustejovsky, J. (2013). Type theory and lexical decomposition. En J. Pustejovsky, P. Bouillon, H. Isahara, K. Kanzaki \& Ch. Lee (Eds.), Advances in Generative Lexicon Theory (pp. 492). Nueva York/Londres: Springer.

Pustejovsky, J. \& Anick, P. (1988). On the semantic interpretation of nominals. Ponencia presentada en el 12th International Conference on Computational Linguistics, Budapest Hungría.

Pustejovsky, J., Bouillon, P., Isahara, H., Kanzaki, K. \& Ch. Lee (Eds.) (2013). Advances in Generative Lexicon Theory. Nueva York/Londres: Springer.

Real Academia Española y Asociación de Academias de la Lengua Española (2009). Nueva gramática de la lengua española. Madrid: Espasa-Calpe.

Ruimy, N. (2006a). Structuring a domain vocabulary in a general knowledge environment. En LREC 2006 Proceedings [en línea]. Disponible en: http://www.cs.brandeis.edu/ marc/misc/proceedings/lrec-2006/index.htm

Ruimy, N. (2006b). A computational multi-layered italian lexicon for hlt applications. En Proceedings XII EURALEX International Congress, Atti del Congresso Internazionale di Lessicografia, 1, 221-227.

Ruimy, N. (2006c). Merging two ontology-based lexical resources. LREC Proceedings [en línea]. Disponible en: http://www.cs.brandeis.edu/ marc/misc/proceedings/lrec-2006/index.htm.

Sánchez Lancis, C. (2016). De la síntesis al análisis: Diacronía del quesuismo en español. En É. Buchi, J.-P. Chauveau \& J.-M. Pierrel (Eds.), Actes du XXVIIe Congrès de linguistique et philologie romanes (pp. 15-20). Section 4: Syntax. Nancy, ATILF.

Sanfelici, E. \& Poletto, C. (2017). Relative clauses. En A. Dufter \& E. Stark (Eds.), Manual of Romance Morphosyntax and Syntax. Berlín /Boston: Walter de Gruyter.

Stallard, D. (1989). Unification-based semantic interpretation in the BBN Spoken Language System. En Proceedings of the workshop on Speech and Natural Language (pp. 39-46). California: Morgan Kaufmann [en línea]. Disponible en: https://dl.acm.org/citation.cfm?id=1075527\&picked=prox

Stark, E. (2009). Romance restrictive relative clauses between macrovariation and universal structures. Philologie im Netr, 47, 1-15. [en línea]. Disponible en: http://web.fu-berlin.de/phin/phin47/p47t1.htm

Stark, E. (2016). Relative clauses. En A. Ledgeway \& M. Maiden (Eds.), The Oxford Guide to the Romance Languages (pp. 1029-1040). Oxford: Oxford University Press. 
Staroverov, P. (2007). Relational nouns and reciprocal plurality. En T. Friedman \& M. Gibson (Eds.), Proceedings of the 17th Semantics and Linguistic Theory conference (pp. 300-316). Nueva York: Cornell University.

Zagona, K. (2002). Sintaxis generativa del español. Madrid: Visor Libros.

Zubizarreta, M. L. (1999). Las funciones informativas: Tópico y foco. En I. Bosque \& V. Demonte (Eds.), Gramática descriptiva de la lengua española (pp. 4215-4244). Madrid: Espasa-Calpe.

\section{NOTAS}

1 En lo sucesivo, adopto la siguiente convención tipográfica: (a) resalto en cursiva el pronombre relativo, grupo relativo o adverbio relativo que, desprovisto de marcas de caso sintáctico, introduce la oración subordinada; (b) resalto en negrita los elementos que transmiten caso sintáctico explícito, como los grupos relativos con preposición, los pronombres reasuntivos o los SDs reasuntivos; y (c) subrayo con punteado los antecedentes, de ser expresos (i. e. el sintagma nominal para las oraciones de relativo explicativas y el núcleo nominal para las especificativas).

${ }^{2}$ En palabras de Pustejovsky (1991: 411): “semantic interpretation proceeds in a principled fashion, always aware of what the source of a particular inference is, and what the certainty of its value is. Such an approach allows the reasoning process to be both tractable and computationally efficient. The representation of lexical semantics, therefore, should be seen as just one of many levels in a richer characterization of contextual structure".

${ }^{3}$ No debe olvidarse, pues, que "The generative approach to lexical semantics derives its name from the use of generative devices instead of a fixed set of primitives. Much of the theory consists of structuring and integrating a number of well-known proposals on specific topics in lexical semantics and knowledge representation into one coherent theory" (Heylen, 1995: 129). Vid. De Miguel $(2012,2014)$ para un panorama actual sobre algunos proyectos lexicológicos en marcha.

${ }^{4}$ En aras de fidelidad al original, cito a continuación las palabras del autor: "By defining the functional behavior of lexical items at different levels of representation we hope to arrive at a characterization of the lexicon as an active and integral component in the composition of sentence meanings. Because of the more expressive mechanisms involved in composition, this approach will enable us to conflate different word senses into a single meta-entry" (Pustejovsky, 1998: 62).

${ }^{5}$ El símil entre los qualia y los sintagmas, por un lado, y entre los mecanismos generativos y las reglas transformacionales, por otro, se encuentra formulado en Pustejovsky (1998: 76): "These qualia are in fact structures very much like phrasal structural descriptions for syntactic analyses, 
which admit of something similar to transformational operations in order to capture polymorphic behavior as well as sense creation phenomena".

${ }^{6}$ Como me hace notar Elena de Miguel (c. p.), contrastes similares de gramaticalidad pueden apreciarse para los ejemplos $\mathrm{Al}$ ordenador se le ha estropeado el cargador / *Al cargador se le ha estropeado el ordenador.

7 Adviértase que el ejemplo (15c) debe interpretarse como una oración de relativo con reasunción, independiente, por tanto, de las denominadas relativas cortadoras (Caviglia \& Malcuori, 2007), no pronominales (RAE \& ASALE, 2009) o pertenecientes a la estrategia vacío de Keenan y Comrie (1977), que resultan de la ausencia de marcas de caso en el pronombre relativo (v. gr. Es una amiga que no te puedes fiar). En este tipo de oraciones de relativo, el complementante que que las introduce está desprovisto de las marcas de caso que, en la estrategia canónica de relativización del español, corresponden al pronombre relativo. Así, el ejemplo (15c), si se interpreta como oración de relativo no pronominal, exige la recuperación de la preposición en para su interpretación (i. e. 'es un libro en el que los epígrafes no tienen sentido'). No es esta, sin embargo, la interpretación que se plantea aquí para el ejemplo (15c).

8 A este respecto, Eschenbach (1993: 3) advierte que: "In addition to the cases of pure predicative nouns and pure relational nouns, there are many nouns which cannot be classified out of context within this dichotomy. Nouns such as child and picture can occur in both uses. There are nouns which obviously have distinct denotations in their predicative and relational uses (e.g. the child of Edith may refer to an adult). To handle such cases, we could assume two different lexical entries. But in most cases, it is not easy to determine whether or not a noun is relational and what kind of argument(s) should be assumed, e.g. table, house, car, clutch". En el modelo de Pustejovsky (1998), sin embargo, la solución teórica implica asumir que el carácter relacional de un nombre como, por ejemplo, hermano está siempre registrado en su estructura subléxica. Lo mismo sucede con los paradigmas léxico-conceptuales abordados en Pustejovsky y Anick (1988).

9 Agradezco a uno de los revisores anónimos el ejemplo, por un lado, y la observación, por otro, según la cual la propuesta teórica aquí defendida permite explicar la extrañeza o agramaticalidad que suscitan aquellos ejemplos en los que un sustantivo relacional simétrico, saturado por el 'pro' de la Flexión, ejerce como argumento interno del SD reasuntivo.

\section{* AGRADECIMIENTOS}

Este proyecto se ha beneficiado de una Ayuda para el Fomento de la Investigación en Estudios de Máster de la Universidad Autónoma de Madrid. A Elena de Miguel debo el magisterio y la motivación, sin los cuales este trabajo no habría sido posible. Las sucesivas versiones de este trabajo se han beneficiado de los comentarios de Elena de Miguel y de dos revisores anónimos. Los errores que persistan solo a mí son imputables. 